Atonic constipation is to be managed in the older child as in the baby, but in the older child treatment is easier, as you have the child's co-operation. But, usually, these older children want more than exercise and massage to the abdomen, they need a complete change, such as being taken from school and put into a place where they will live an open-air life, so toning up the whole system.

In the cases showing spasm you must give a bland, unirritating diet, because it is sometimes an excess of "roughage" which sets up the spastic condition. These are the children who cannot deal with raw fruit, but they may be able to take cooked fruit and sieved vegetables. And in those, as in infant cases, you give belladonna and perhaps a mild laxative or lubricant, such as paraffin.

Where there is an excess of mucus the diet must be different: it is necessary then to reduce the starches and sugars, particularly cane sugar. To prevent the formation of mucus there is nothing better than the old rhubarb and soda mixture. In the older children, as in infants, you will meet with patients whom you will find it difficult to refer to any one of the categories I have named: cases in which there seems to be nothing definitely wrong with the diet; plenty of opportunity is given for the bowels to act and there is neither bowel spasm, nor excess of mucus in the motions. These you speak of again as simple constipation. Some have said these are due to the colon being abnormally long, and that as the child grows its length will become approximated to the normal. There may be something in that, but such an abnormality would be revealed by X-rays. And, whether there is a redundant colon or not, you must, just as in grown-up people, resort in such cases to aperients. Often parents are reluctant to give their child aperients regularly, but provided the laxative is properly chosen and is given in reasonable doses, no ultimate harm will result: on the contrary, by the proper use of laxatives you can educate the bowels to act regularly, and by and bye the dose can be diminished to a very small one, and finally can be dispensed with entirely. The most suitable aperients are the same as are used for grown-ups. Paraffin is a good lubricant, but usually in the cases I am referring to it is not sufficient. I prefer cascara or senna. Aloes is a good tonic aperient, but it is apt to gripe. I often give elixir of cascara, at bed-time, and children take it readily. Or you may give one of the forms of senna, either syrup of figs, or an infusion of senna pods. You proceed in the same way as for adults, finding the adequate dose and-an important matter-giving it regularly. There is an advantage in varying the kind of aperient: I like to ring the changes with one or two of the tonic laxatives, so as to avoid having to increase the dose.

\title{
CHRONIC EMPYEMATA.*
}

By H. P. NELSON, M.D., F.R.C.S.

Chief Assistant to a Surgical Unit, St. Bartholomew's Hospital; Assistant Surgeon to the Brompton Hospital for Diseases of the Chest.

Chronic empyemata may be either (I) Pyogenic, or (2) Tuberculous.

(I) Pyogenic : (a) Those following the drainage of an acute empyema. An acute empyema becomes chronic when the cavity persists for an abnormal length of time after the original drainage. It may either be draining more or less completely through the opening in the chest wall, or, the opening having been allowed to heal, pus has reaccumulated in the cavity.

*Post Graduate Lecture at the Brompton Hospital on 25th October, 1933. 
A chronic empyema rarely occurs if the original acute empyema has been treated properly. There are many different opinions as to the proper treatment for an acute empyema, but little difference in the final result if two main principles are observed, namely, drainage at the correct time and continued until the cavity is completely obliterated.

As to the correct time, drainage should never be performed during the acute pneumonic process, but delayed until the pleural adhesions round the margins of the empyema are sufficiently strong to prevent collapse of the whole lung and a shift of the mediastinum when the chest is opened. Drainage performed too early may cause the death of the patient, but, on the other hand, if delayed too long there will be a thick fibrous deposit on the visceral pleura which will hamper the lung in its expansion. The optimum time can be estimated from a sample of pleural fluid after it has stood in a test-tube for twenty-four hours; the pus will settle to the bottom, leaving the serum above, and when there is 75 to 80 per cent. of pus, open drainage may be performed with safety. Closed intercostal drainage is employed in infants and, very occasionally, in synpneumonic empyemata, but is unsatisfactory for the ordinary meta-pneumonic empyemata.

By far the commonest cause of a chronic empyema is the removal of the drainage-tube before the cavity is obliterated.

If the tube is removed while the cavity is still discharging pus, the opening in the chest wall, although reduced to a pinhole, is kept open by the pressure of the pus in the chest; if, on the other hand, the tube is not removed until the cavity is "clean," the opening in the chest wall closes rapidly. What happens to the cavity after closure of the drainage opening must depend on the number and virulence of any remaining organisms and the resistance of the host. In the majority of cases the tissues are able to deal with the infection, the lung gradually expands and the cavity is obliterated by firm fibrous union between the two pleural surfaces. But in others a small pocket or chink is left that contains micro-organisms, and sooner or later these get the upper hand, pus accumulates and distends the cavity and the patient becomes ill again; the empyema then either ruptures spontaneously through the old site of drainage, or is redrained by operation. Too often the doctor, not appreciating what has happened, again removes the tubes too early; the same story is then repeated time and time again, and in the meanwhile the lung is becoming imprisoned by a thicker and thicker layer of fibrous tissue, so that closure of the cavity becomes impossible without an extensive operation. It is surprising how long an interval of apparently normal health can intervene before these empyemata recur. I have known cases go from ten to fifteen years, and then get a recurrence at the site of the previous empyema ; this would be impossible if the original empyema had been completely healed.

There are three simple methods of examining an empyema cavity to find out its size, and to know when it is obliterated, viz.: (a) The introduction of a finger through the drainage opening; $(b)$ probing the cavity through the drainage opening with a uterine sound, soft metal gall-stone probe, or urethral bougie; $(c)$ measuring the capacity with fluid as follows: The patient lies down so that the opening is the highest point of the cavity, and fluid is then slowly introduced with a graduated syringe; this method cannot be carried out so long as there is a broncho-pleural fistula.

The drainage tube should have its inner end just inside the cavity, and be fixed in that position by a safety-pin and adhesive strapping attached to the chest wall, so that it will not slip in or out. It is unnecessary to take the tube out more than once a 
week, and instead of daily irrigations (mechanical lavage) it is preferable to lay the patient down and then fill the cavity with Dakin's solution and leave the patient in that position for fifteen minutes. When the cavity is clean and its capacity is under 25 c.c. the size of the drainage tube is reduced, and finally a small soft capillary tube remains until the last traces of the cavity are obliterated. In the ordinary course of events an empyema should be completely healed in four to six weeks.

The longer the walls of an empyema cavity are bathed in pus, the thicker becomes the fibrous deposit on the visceral pleura, and therefore after drainage, the quicker the infection can be cleared up the better is the chance of an early expansion of the lung. There are several points to be noted in this connection: (a) The removal at the time of operation of all fibrin lying loose in the cavity or adherent to its walls. (b) The avoidance of a puddle of pus at the bottom of the cavity by having the drainage opening at the lowest point. (c) The avoidance of a long drainage tube projecting up into the cavity so that pus can only escape by overflow, which state of affairs is indicated if a stream of pus escapes when the tube is withdrawn. (d) The use of Dakin's solution, which assists in clearing up the infection.

$A$ foreign body in an empyema may prevent it from healing, or it may appear to heal, only to break down again at some later date. One of the commonest types of foreign body is a portion of drainage tube, or dressing. This can be prevented if there is a safety-pin through the extra-thoracic portion of the tube, and the pin is fixed to the skin with adhesive strapping. Another type of foreign body is a piece of dead bone, which is the result of osteo-myelitis of the cut ends of the ribs. These are not seen on an X-ray, and are only discovered when the cavity is re-explored at operation.

Finally, there is a group of causes quite unavoidable by the surgeon that lie in the nature of the infecting organism, or an abnormal atiology which may have been unsuspected at the time of drainage. A secondarily infected tuberculous empyema is not readily distinguished from an ordinary pyogenic one unless there is a previous history that throws light on the diagnosis. These are drained, quite correctly, by open operation, and it is only when the lung fails to expand that the underlying tuberculous infection is suspected. Sometimes the reverse happens, namely a secondary tuberculous infection in a chronic pyogenic empyema. If the diagnosis is in doubt at the time of drainage, a biopsy should be taken from the parietal pleura. Sections are cut from one piece, while the rest is ground into an emulsion and injected into a guinea-pig. Actinomycosis very occasionally causes an empyema, and in bronchial carcinoma an empyema develops in Io per cent. of cases. In both of these conditions the pus must be drained, but unless they have been fully investigated beforehand, the underlying cause will not be discovered until the time of operation or later.

If an ordinary empyema is still discharging pus after three weeks, and is not healed at the end of six, investigation should be carried out to find the reason for this delay. The history should be reviewed for a possible unsuspected ætiology, such as tubercle or growth. The drainage tube and cavity should be examined to see that the drainage is satisfactory, the size and capacity of the remaining cavity should be measured, and the inside of the cavity may even be inspected with a cystoscope or bronchoscope. Some of the granulated tissue lining the cavity should be removed with a curette for investigation. X-rays are employed to show the state of the lung parenchyma, to exclude a loculated collection of pus, and confirm the clinical observations as to the site of drainage and size of the cavity. The cavity can be best demonstrated by filling it with an opaque medium, and then taking antero-posterior and lateral films. Lipiodol or barium emulsion are the usual sub- 
stances, the cavity being filled with the patient lying down, and the opening is then plugged with gauze to prevent its escape. If a fistula is present the lipiodol is injected into a long piece of fine rubber tubing, which is then pushed into the cavity, where it coils round the walls.

I do not wish to go into the treatment of these chronic empyemata, but merely to say that if after thorough and prolonged drainage, with breathing exercises, etc., the lung cannot be brought out to the chest wall, then an operation will be required to bring the chest wall down to the lung. There are, however, a few exceptional cases, such as those occurring in elderly people, when it is wisest to leave the patient with a drainage tube for the rest of his life.

(b) Latent empyemata, namely those in which the original acute empyema has been overlooked. In these cases the empyema has either become inspissated and the patients show signs of a chronic toxæmia, or the empyema has ruptured into a bronchus and the patient is coughing it up, when it may be mistaken for a case of lung abscess or bronchiectasis. These cases require thorough drainage for a long time, but in the majority the lung fails to come out to the chest wall and further operative procedures are necessary.

(2) Tuberculous : (a) Clear Effusions. Although these are not chronic empyemata, it may be as well to consider them here. They may complicate pneumothorax treatment, or may develop spontaneously. They can be divided into two groups: those in which no tubercle bacilli can be found in a centrifuged deposit, and those which contain tubercle bacilli. The former group may well be left alone, excepto when the patient is embarrassed on account of the size of the effusion; but the latter group, where the tubercle bacilli are present, if left alone usually go on to the formation of a thick tuberculous pus. They are best treated by aspiration to keepa the pleural cavity as dry as possible.

(b) Pure Tuberculous Empyemata, i.e., those in which the pus is sterile on culture. In practically all these cases the object of therapy should be to encourage expansion of the lung to obliterate the pleural cavity. This is best performed by aspiration and pleural washouts as follows:-

The patient is put in a sitting-up position. A large trocar and cannula is inserted into the bottom of the empyema as near the front of the chest as possible. An artificial pneumothorax needle is inserted at the top of the empyema, usually in the second interspace in front. The cannula is connected with a Potain's aspiration bottle, and as the empyema is evacuated air enters through the upper needle. The cannula is then connected by rubber tubing with a funnel, and half-strength Dakin's, warmed to body temperature, is run in until it starts to escape from the upper needle. The cannula is then connected again with the aspiration bottle and the cavity emptied. This process is repeated three or more times until the returning fluid is clear. The cannula is then withdrawn and the upper needle is connected with a pneumothorax apparatus, by means of which the air in the cavity is evacuated, leaving it at a negative tension. These pleural washouts may be repeated at weekly intervals and are continued until the X-ray shows that the expansion of the lung is at a standstill. If it has not come out completely a thoracoplasty will be necessary to obliterate the pleural space.

(c) Secondarily Infected Tuberculous Empyemata. These cases must be treated like ordinary acute empyemata, and be drained with a tube. The outlook for them, however, is extremely bad, and there is a mortality rate in the region of 80 per cent. Should they survive the infection, they must either continue with a drainage tube or have a thoracoplasty to obliterate the space. 